\title{
ALTERNATIVE TO FOUR-COMPONENT DECOMPOSITION FOR POLARIMETRIC SAR
}

\author{
J. X. Zhang, G. M. Huang, J. J. Wei*, Z. Zhao \\ Institute of Photogrammetry and Remote Sensing, Chinese Academy of Surveying \& Mapping, Beijing 100830, P.R. China; \\ E-Mail: zhangjx@casm.ac.cn; huang.guoman@163.com; zhengzhaochina@163.com;
}

\section{Commission VII, WG VII/6}

KEY WORDS: Four-component decomposition, Generalized similarity parameter (GSP), Eigenvalue decomposition, Polarimetric SAR (PolSAR)

\begin{abstract}
:
There are more unknowns than equations to solve for previous four-component decomposition methods. In this case, the nonnegative power of each scattering mechanism has to be determined with some assumptions and physical power constraints. This paper presents a new decomposition scheme, which models the measured matrix after polarimetric orientation angle (POA) compensation as a linear sum of five scattering mechanisms (i.e., odd-bounce scattering, double-bounce scattering, diffuse scattering, volume scattering, and helix scattering). And the volume scattering power is calculated by a slight modified NNED method, owing to this method considering the external volume scattering model from oblique dihedral structure. After the helix and volume scattering powers have been determined sequentially, the other three scattering powers are estimated by combining the generalized similarity parameter (GSP) and the eigenvalue decomposition. Among them, due to POA compensation, the diffuse scattering induced from a dihedral with a relative orientation of $45^{\circ}$ has negligible scattering power. Thus, the new method can be reduced as four-component decomposition automatically. And then the ALOS-2 PolSAR data covering Guiyang City, Guizhou Province, China were used to evaluate the performance of the new method in comparison with some classical decomposition methods (i.e. Y4R, S4R and G4U).
\end{abstract}

\section{INTRODUCTION}

Polarimetric target decomposition plays an important role in the interpretation of polarimetric SAR (POLSAR) data. Freeman and Durden (1998) firstly proposed the original threecomponent decomposition, modeling the measured covariance matrix as a linear sum of three physical scattering mechanisms (i.e., surface scattering, double-bounce scattering, and volume scattering) under the reflection symmetry condition. Then Yamaguchi et al. (2005) extends the three-component decomposition method by adding helix scattering mechanism as the fourth component for non-reflection symmetric scattering case. But there exist a fatal flaw for the two methods in that negative powers will appear in the image analysis. Thus, Yajima et al. (2008) modified the Yamaguchi decomposition approach by taking into account physical conditions to overcome the deficiency. Additionally, there are more unknowns than equations to solve for the existing model-based decomposition (Freeman and Durden, 1998; Yamaguchi et al., 2005; Yajima et al., 2008; An et al., 2010; Yamaguchi et al., 2011; Sato et al., 2012; Singh et al., 2013). In order to determine the powers of the surface and double-bounce scattering mechanisms, the methods should suppose the magnitude of the unknown parameter $\alpha$ in the double-bounce scattering model to be -1 if $\operatorname{Re}\left(S_{\mathrm{HH}} S_{\mathrm{VV}}^{*}\right)>0$ or suppose the unknown parameter $\beta$ in the surface scattering model equal to +1 if $\operatorname{Re}\left(S_{\mathrm{HH}} S_{\mathrm{VV}}^{*}\right)<0$. Since the parameters $\alpha$ and $\beta$ are separately assumed to be -1 and +1 , the corresponding scattering models of the Pauli spin matrices separately representing odd-bounce scattering, double-bounce scattering and diffuse scattering, are directly applied to the proposed decomposition method in this paper. The proposed

\footnotetext{
* Corresponding author: E-Mail: weijujie0417@163.com
}

method models the covariance matrix after polarimetric orientation angle (POA) compensation (Lee and Ainsworth, 2011) as a linear sum of five scattering mechanisms (i.e., oddbounce scattering, double-bounce scattering, diffuse scattering, volume scattering, and helix scattering). Then the helix scattering power is estimated by the same way to the existing four-component decomposition. After that, the volume scattering power is determined by a slightly modified nonnegative eigenvalue decomposition (NNED) method. Finally, the other three scattering powers (i.e., odd-bounce scattering, double-bounce scattering and diffuse scattering) are determined by the combination of the generalized similarity parameter (GSP) (An et al., 2009) and the eigenvalue decomposition (Cloude and Pottier, 1996). Due to POA compensation, the diffuse scattering induced from a dihedral with a relative orientation of $45^{\circ}$ has negligible scattering power. In this context, the five-component decomposition can be reduced as four-component decomposition automatically. Furthermore, because the GSP is always greater than or equal to zero, the new method also can avoid negative powers in decomposition results.

\section{GENERALIZED SIMILARITY PARAMETER}

The GSP is an extension to the similarity parameter (Yang et al., 2001) for multi-look POLSAR data and is defined as

$$
R\left(\mathbf{T}_{1}, \mathbf{T}_{2}\right)=\frac{\left|\left\langle\mathbf{T}_{1}, \mathbf{T}_{2}\right\rangle\right|}{\left\|\mathbf{T}_{1}\right\|_{\mathrm{F}}\left\|\mathbf{T}_{2}\right\|_{\mathrm{F}}}=\frac{\left|\operatorname{tr}\left(\mathbf{T}_{1}^{\dagger} \mathbf{T}_{2}\right)\right|}{\sqrt{\operatorname{tr}\left(\mathbf{T}_{1}^{\dagger} \mathbf{T}_{1}\right) \cdot \operatorname{tr}\left(\mathbf{T}_{2}^{\dagger} \mathbf{T}_{2}\right)}}
$$

where the matrices $\mathbf{T}_{1}$ and $\mathbf{T}_{2}$ correspond to the coherence or covariance matrix; $\operatorname{tr}(\cdot)$ is the trace of a matrix; $\langle\cdot\rangle$ is the inner 
product of two matrices, defined as $\left\langle\mathbf{T}_{1}, \mathbf{T}_{2}\right\rangle=\operatorname{tr}\left(\mathbf{T}_{1}^{\dagger} \mathbf{T}_{2}\right)$; the superscript $\uparrow$ represents the conjugate transpose of a matrix; and $\|\cdot\|_{F}$ is the Frobenius norm of a matrix.

As the mention in An et al. (2009), the GSP has some important properties as follows:

(1) The GSP is always greater than or equal to zero;

(2) If $\mathbf{T}_{1}, \mathbf{T}_{2}$, and $\mathbf{T}_{3}$ are three non-zero coherence or covariance matrices, which satisfy

$$
R\left(\mathbf{T}_{1}, \mathbf{T}_{2}\right)=R\left(\mathbf{T}_{1}, \mathbf{T}_{3}\right)=R\left(\mathbf{T}_{2}, \mathbf{T}_{3}\right)=0
$$

in that the matrices $\mathbf{T}_{1}, \mathbf{T}_{2}$ and $\mathbf{T}_{3}$ are mutually orthogonal, then for an arbitrary coherence matrix $\mathbf{T}$, with the rank of one, we have

$$
R\left(\mathbf{T}, \mathbf{T}_{1}\right)+R\left(\mathbf{T}, \mathbf{T}_{2}\right)+R\left(\mathbf{T}, \mathbf{T}_{3}\right)=1
$$

\section{NEW DECOMPOSITION SCHEME}

\subsection{Basic decomposition principle}

The proposed method models the covariance matrix $\langle\mathbf{C}\rangle$ after POA compensation as a linear sum of five scattering mechanisms (i.e., odd-bounce scattering, double-bounce scattering, diffuse scattering, volume scattering, and helix scattering) as follows

$$
\langle\mathbf{C}\rangle=f_{\text {odd }} \mathbf{C}_{\text {odd }}+f_{\text {dbl }} \mathbf{C}_{\mathrm{dbl}}+f_{\text {diff }} \mathbf{C}_{\text {diff }}+f_{\mathrm{vol}}\langle\mathbf{C}\rangle_{\mathrm{vol}}+f_{\mathrm{hlx}} \mathbf{C}_{\mathrm{hlx}}
$$

where $f_{\text {odd }}, f_{\text {dbl }}, f_{\text {diff }}, f_{\text {vol }}$ and $f_{\text {hlx }}$ are the scattering powers to be determined. And $\mathbf{C}_{\text {odd }}, \mathbf{C}_{\mathrm{dbl}}, \mathbf{C}_{\mathrm{diff}},\langle\mathbf{C}\rangle_{\mathrm{vol}}$, and $\mathbf{C}_{\mathrm{hlx}}$ corresponds to the known scattering mechanisms, i.e., oddbounce scattering, double-bounce scattering, diffuse scattering, volume scattering, and helix scattering, respectively.

For the existing model-based decomposition methods, the odd\& double-bounce scattering models are expressed as (5), respectively.

$$
\mathbf{C}_{\text {odd }}=\left[\begin{array}{ccc}
|\beta|^{2} & 0 & \beta \\
0 & 0 & 0 \\
\beta^{*} & 0 & 1
\end{array}\right], \mathbf{C}_{\mathrm{dbl}}=\left[\begin{array}{ccc}
|\alpha|^{2} & 0 & \alpha \\
0 & 0 & 0 \\
\alpha^{*} & 0 & 1
\end{array}\right]
$$

where $\alpha$ and $\beta$ are the unknown parameters to be determined. And the superscript asterisk $(*)$ represents complex conjugate.

Since the parameters $\alpha$ and $\beta$ have to be separately supposed to be -1 and +1 for the determination of the odd- \& doublebounce scattering powers, the corresponding covariance matrices of the Pauli spin matrices (Lee and Pottier, 2009) are directly applied for the proposed method in this paper, as follows

$$
\mathbf{C}_{\text {odd }}=\frac{1}{2}\left[\begin{array}{lll}
1 & 0 & 1 \\
0 & 0 & 0 \\
1 & 0 & 1
\end{array}\right], \mathbf{C}_{\mathrm{dbl}}=\frac{1}{2}\left[\begin{array}{rcc}
1 & 0 & -1 \\
0 & 0 & 0 \\
-1 & 0 & 1
\end{array}\right], \mathbf{C}_{\mathrm{diff}}=\left[\begin{array}{lll}
0 & 0 & 0 \\
0 & 1 & 0 \\
0 & 0 & 0
\end{array}\right](6)
$$

where $\mathbf{C}_{\text {diff }}$ represents a dihedral with a relative orientation of $45^{\circ}$, named diffuse scattering here.

Moreover, as mentioned in Sato et al. (2012), the volume scattering model $\langle\mathbf{C}\rangle_{\text {vol }}$ also accounts for the HV component for dihedral structures besides vegetation to distinguish oriented urban areas from vegetation. And the helix scattering model $\mathbf{C}_{\mathrm{hlx}}$ is expressed as the same to the mention in the references (Yamaguchi et al., 2005; Yajima et al., 2008; Yamaguchi et al., 2011; Sato et al., 2012; Singh et al., 2013).

\subsection{Determination of the scattering powers}

The powers of the five scattering components are determined in the following order.

\section{(1) Helix scattering contribution}

The helix scattering contribution is determined as the same to the existing four component decomposition, in that

$$
f_{\text {hlx }}=2\left|\operatorname{Im}\left(T_{23}\right)\right|
$$

(2) Volume scattering contribution

If the helix scattering is determined, the remainder matrix $\left\langle\mathbf{C}_{\text {remainder }}^{1}\right\rangle$ can be obtained after the helix component have been subtracted from the measurable data and is expressed as

$$
\left\langle\mathbf{C}_{\text {remainder }}^{1}\right\rangle=a\left\langle\mathbf{C}_{\text {vol }}\right\rangle+\left\langle\mathbf{C}_{\text {remainder }}^{2}\right\rangle
$$

where the parameter $a$ is the volume scattering power to be determined.

Van Zyl et al. $(2008$, 2011) proposed NNED method to determine the volume scattering power, which is of benefit to avoid negative powers in decomposition results. However, due to the volume scattering model that also accounts for the HV component for dihedral structures in this paper, the NNED is modified slightly to avoid the denominator of a fractional expression from solving the parameter $a$ to be zero in the data processing procedure. Then the parameter $a$ is estimated by

$$
a=\min \left(a_{1}, a_{2}\right)
$$

where $a_{1}=\left\{\begin{array}{cl}\frac{\xi \zeta-|\rho|^{2}}{Z} & \text { if } \xi_{a} \zeta_{a}-\left|\rho_{a}\right|^{2}=0 \\ \frac{Z-\sqrt{Z^{2}-4\left(\xi_{a} \zeta_{a}-\left|\rho_{a}\right|^{2}\right)\left(\xi \zeta-|\rho|^{2}\right)}}{2\left(\xi_{a} \zeta_{a}-\left|\rho_{a}\right|^{2}\right)} & \text { otherwise }\end{array}\right.$, $a_{2}=\eta / \eta_{a}$.

where $\quad \xi=\left\langle\left|S_{H H}\right|^{2}\right\rangle-\frac{1}{4} f_{h l x} \quad, \quad \rho=\left\langle S_{H H} S_{V V}^{*}\right\rangle+\frac{1}{4} f_{h l x} \quad$, $\eta=2\left\langle\left|S_{H V}\right|^{2}\right\rangle-\frac{1}{2} f_{h l x}, \zeta=\left\langle\left|S_{V V}\right|^{2}\right\rangle-\frac{1}{4} f_{h l x}$. And $\xi_{a}, \zeta_{a}, \rho_{a}$, and $\eta_{a}$ are the known parameters in the volume scattering model. 


\section{(3) Other three scattering contribution}

After that the helix and volume scattering powers have been determined, the second remainder matrix $\left\langle\mathbf{C}_{\text {remainder }}^{2}\right\rangle$ only contains odd-bounce scattering, double-bounce scattering, and diffuse scattering. And the three scattering models shown in (6) are mutually orthogonal. But the rank of $\left\langle\mathbf{C}_{\text {remainder }}^{2}\right\rangle$ is not equal to one. In order to utilize the GSP to determine their corresponding scattering contributions, the eigenvalue decomposition is exploited for the remainder matrix $\left\langle\mathbf{C}_{\text {remainder }}^{2}\right\rangle$ to get three different single scatters $\mathbf{C}_{i}$ with its rank of one, as follows

$$
\left\langle\mathbf{C}_{\text {remainder }}^{2}\right\rangle=\sum_{i=1}^{3} \lambda_{i} \vec{u}_{i} \vec{u}_{i}^{\dagger}=\lambda_{1} \mathbf{C}_{1}+\lambda_{2} \mathbf{C}_{2}+\lambda_{3} \mathbf{C}_{3}
$$

where $\lambda_{i}$ is the real eigenvalue of the matrix and $\vec{u}_{i}$ is the corresponding eigenvector.

Then the GSP is exploited to determine the three scattering contributions. By combining the properties of the GSP (2) (3) with the decomposed results (10), we can get

$$
\begin{gathered}
R\left(\mathbf{C}_{i}, \mathbf{C}_{\text {odd }}\right)+R\left(\mathbf{C}_{i}, \mathbf{C}_{\mathrm{dbl}}\right)+R\left(\mathbf{C}_{i}, \mathbf{C}_{\text {diff }}\right)=1 \\
\lambda_{i}=\lambda_{i}\left\{R\left(\mathbf{C}_{i}, \mathbf{C}_{\text {odd }}\right)+R\left(\mathbf{C}_{i}, \mathbf{C}_{\mathrm{dbl}}\right)+R\left(\mathbf{C}_{i}, \mathbf{C}_{\text {diff }}\right)\right\}
\end{gathered}
$$

Then the three scattering powers can be determined as

$$
\begin{gathered}
f_{\text {odd }}=\sum_{i=1}^{3} \lambda_{i} R\left(\mathbf{C}_{i}, \mathbf{C}_{\text {odd }}\right) \\
f_{\mathrm{dbl}}=\sum_{i=1}^{3} \lambda_{i} R\left(\mathbf{C}_{i}, \mathbf{C}_{\mathrm{dbl}}\right) \\
f_{\text {diff }}=\sum_{i=1}^{3} \lambda_{i} R\left(\mathbf{C}_{i}, \mathbf{C}_{\text {diff }}\right)
\end{gathered}
$$

\section{EXPERIMENTAL RESULTS}

\subsection{Algorithm verification}

In this section, many experiments were performed to evaluate the performance of the new method in comparison with classical decomposition methods (i.e. Y4R (Yamaguchi et al., 2011), S4R (Sato et al., 2012) and G4U (Singh et al., 2013)) using ALOS-2 PolSAR data covering Guiyang City, Guizhou Province, China (See Figure 1). And the main acquisition parameters of the data are listed in Table 1 . The PolSAR data were processing with sliding widow of size $5 \times 5$ pixels. Then the decomposition results from the new method are shown in Figure 2.

\begin{tabular}{|l|c|}
\hline Key Parameters & Values \\
\hline Acquisition time & $2015-10-28$ \\
Wavelength $(\mathrm{m})$ & 0.2424525 \\
Azimuth spacing $\Delta \rho_{a}(\mathrm{~m})$ & 9.339 \\
Slant range spacing $\Delta \rho_{r}(\mathrm{~m})$ & 5.722 \\
Looks number in azimuth & 3 \\
Looks number in range & 2 \\
Pass & Descending \\
Antenna pointing & Right \\
Heading angle $\left({ }^{\circ}\right)$ & -168.532 \\
\hline
\end{tabular}

Table 1. The main acquisition parameters

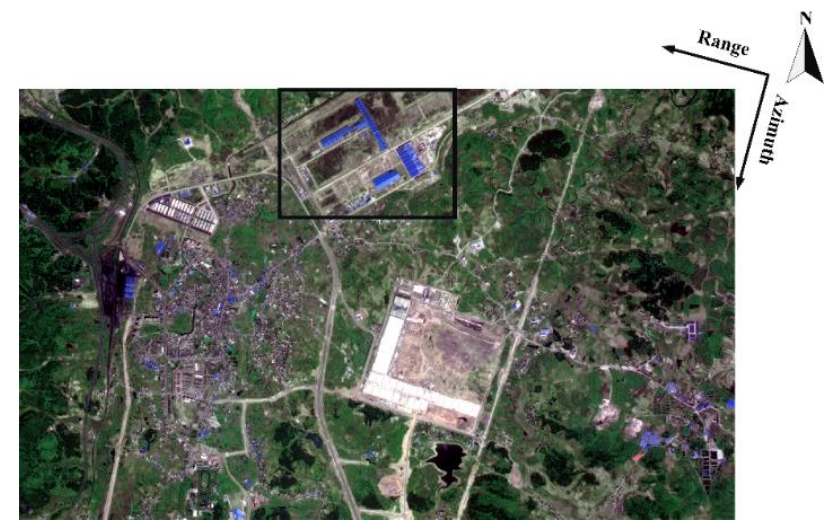

Figure 1. Optical image (Acquisition time: Jun. 2015)
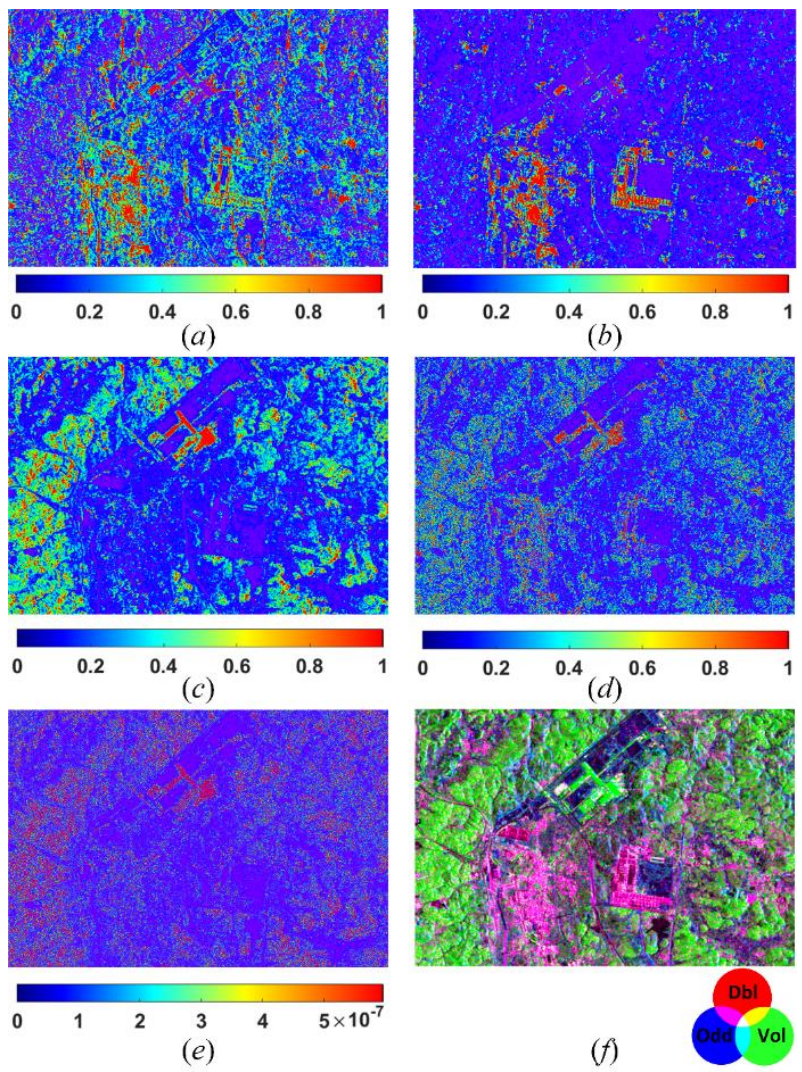

Figure 2. The decomposition results by the new method. (a)

Odd-bounce scattering; (b) Double-bounce scattering; $(c)$

Volume scattering; (d) Helix scattering; (e) Diffuse scattering; $(f)$

RGB image color coded by red: double-bounce (Dbl), green:

volume scattering (Vol), blue: odd-bounce (Odd).

It is seen in Figure $2(f)$ that green is rather strong for the areas covered by forest or vegetation where the volume scattering is predominant and red is especially strong in some urban areas (e.g. center scene). The red area has a common feature that the orientation of building blocks is almost parallel or orthogonal to the SAR flight path. And for the urban areas, it appears a mixture scattering of odd-bounce (for building walls or floors) and double-bounce (for the structure of ground-wall) (See Figures 2(a) and $(b))$. Moreover, the blue area corresponds to relatively level surface, where the odd-bounce scattering 
mechanism is predominant. By comparing Figures 1 and 2, the decomposition result is reasonable. In addition, the skew buildings outlined by the black rectangle in Figure 1 produce a rather dominant volume scattering and appear strong helix scattering power (See Figures $2(c)$ and $(d)$ ). And Figure 2(e) shows the diffuse scattering mechanism corresponds to additional cross-polarized power generated from terrain effects and rough surface. But the magnitude of the diffuse scattering power is about $5 \times 10^{-7}$, which is so small that it can be ignored in the decomposed results. In this case, the diffuse scattering mechanism can be ignored so that the new method can be reduced as four-component decomposition automatically. Thus, the new method can work as an alternative to four-component decomposition.

\subsection{Discussion}

The advanced four-component decomposition methods (i.e. Y4R, S4R, and G4R) were also applied to this PolSAR data. Then the decomposed images are shown in Figure 3.

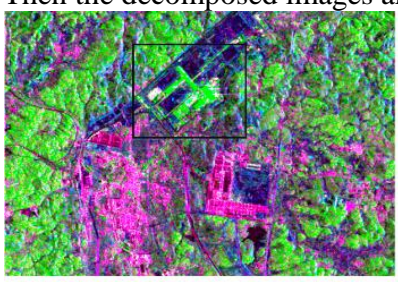

(a)

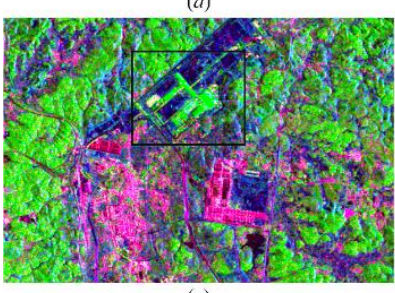

(c)

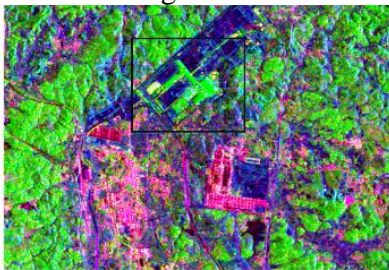

(b)

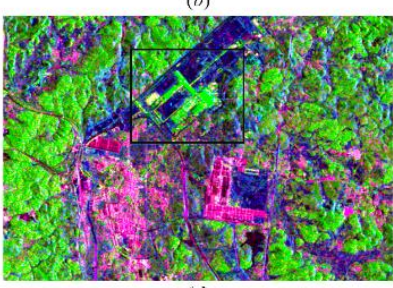

(d)

Figure 3. The RGB image color coded by red: double-bounce, green: volume scattering, blue: odd-bounce, from the four methods. (a) the new method; (b) Y4R; (c) S4R; (d) G4U.

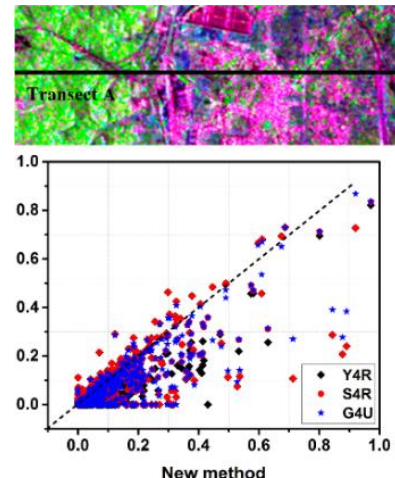

(a)

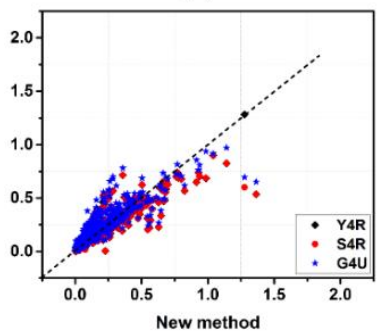

(c)

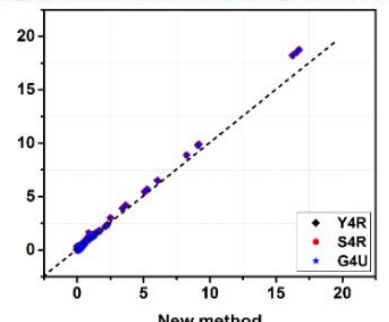

(b)

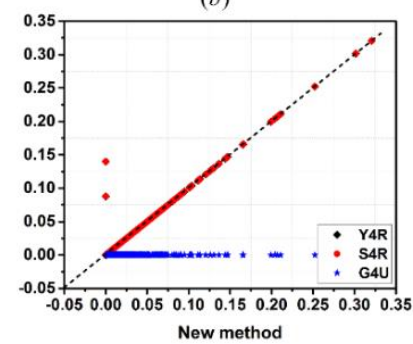

(d)
Figure 4. The quantitative comparison among the four methods. (a) Odd-bounce scattering; (b) Double-bounce scattering; (c) Volume scattering; (d) Helix scattering.

Compared with Figures 3(b)- $(d)$, Figure 3(a) shows that our method can get similar decomposition results to the three methods. For Y4R method, it minimizes the cross-polarized (HV) scattering power generated from dipole by rotation of the measured matrix to discriminate vegetation from oblique urban areas. And the new method also minimizes the cross-polarized scattering power generated from dipole and dihedral as the same to both the methods (S4R and G4U) to further discriminate vegetation from oblique urban. But the skew building outlined by the black rectangle in Figure 3 still appears rather dominant volume scattering mechanism.

Besides, the transect A shown in Figure 4 was chose for the quantitative comparison of our method with the three methods. And Figure 4 shows that the power of each scattering component from the new method is approximatively linearly proportional to that of the corresponding scattering component from the three methods. But the helix scattering contribution of the proposed method is different from that of G4U method sometimes (See Figure 4(d)). It may be caused by the different branch condition used for selecting the dihedral volume scattering model (i.e. the branch condition is $C_{1}=T_{11}(\theta)-T_{22}(\theta)+\frac{7}{8} T_{33}(\theta)+\frac{1}{16} f_{\mathrm{hlx}} \quad$ for $\quad \mathrm{G} 4 \mathrm{U}, \quad$ and $C_{1}=T_{11}(\theta)-T_{22}(\theta)-\frac{1}{2} f_{\mathrm{hlx}}$ for the proposed method as the same to S4R). In a short, the new method can yield similar decomposition results to the existing four-component decomposition

\section{CONCLUSION}

This paper presents a new decomposition scheme, which models the measured matrix after POA compensation as a linear sum of five scattering mechanisms (i.e., odd-bounce scattering, double-bounce scattering, diffuse scattering, volume scattering, and helix scattering). Because the proposed method also minimize the cross-polarized (HV) scattering power generated from dipole plus dihedral, the magnitude of the diffuse scattering mechanism accounting for additional cross-polarized power generated from terrain effects and rough surface is so small that it can be ignored. Thus, our method can be reduced as four-component decomposition automatically. And by experiments, it is proved that this method can yield similar decomposition results to the existing four-component decomposition and can work as an alternative method to fourcomponent decomposition.

In addition, the skew building with respect to the radar direction of illumination still appears rather dominant volume scattering mechanism even though the new method besides S4R and G4U minimizes the cross-polarized scattering power generated from dipole plus dihedral. Therefore, in order to distinguish vegetation from oblique urban areas within the volume scattering mechanism for more accurate classification, there is still lots of work for us to do in our future research. 


\section{ACKNOWLEDGEMENTS}

The study was financially supported by the Special Fund by Surveying \& Mapping and Geoinformation Research in the Public Interest (201412002).

\section{REFERENCES}

An, W. T., Zhang, W. J., and Yang, J., et al., 2009. On the similarity parameter between two targets for the case of multilook polarimetric SAR. Chinese Journal of Electronics, 18(3), pp. $545-550$.

An, W. T., Cui, Y., and Yang, J., 2010. Three-component model-based decomposition for polarimetric SAR data. IEEE Trans. Geosci. Remote Sens., 48(6), pp. 2732 - 2739.

Cloude, S. R., and Pottier, E., 1996. A review of target decomposition theorems in radar polarimetry. IEEE Trans. Geosci. Remote Sens., 34(2), pp. 498 - 518.

Freeman, A., and Durden, S. L., 1998. A three-component scattering model for polarimetric SAR data. IEEE Trans. Geosci. Remote Sens., 36(3), pp. 963 - 973.

Lee, J. S., and Pottier, E., 2009. Polarimetric radar imaging: from basics to applications. Taylor \& Francis Group, Boca Raton, pp. $179-264$.

Lee, J. S., and Ainsworth, T. L., 2011. The effect of orientation angle compensation on coherency matrix and polarimetric target decompositions. IEEE Trans. Geosci. Remote Sens., 49(1), pp. $53-64$.

Sato, A., Yamaguchi, Y., and Singh, G., et al., 2012. Fourcomponent scattering power decomposition with extended volume scattering model. IEEE Geosci. Remote Sens. Lett., 9(2), pp. $166-170$.

Singh, G., Yamaguchi, Y., and Park, S. E., 2013. General fourcomponent scattering power decomposition with unitary transformation of coherency matrix. IEEE Trans. Geosci. Remote Sens., 51(5), pp. 3014 - 3022.

Van Zyl, J. J., Yunjin, K., and Arii, M., 2008. Requirements for model-based polarimetric decompositions. In: IEEE International Geoscience and Remote Sensing Symposium, Boston, MA, Vol. 5, pp. V-417 - V-420.

Van Zyl, J. J., Arii, M., and Yunjin, K., 2011. Model-based decomposition of polarimetric SAR covariance matrices constrained for nonnegative eigenvalues. IEEE Trans. Geosci. Remote Sens., 49(9), pp. $3452-3459$.

Yang, J., Peng, Y. N., and Lin, S. M., 2001. Similarity between two scattering matrices. Electronics Letters, 37(3), pp. 193 194.

Yamaguchi, Y., Moriyama, T., and Ishido, M., et al., 2005. Four-component scattering model for polarimetric SAR image decomposition. IEEE Trans. Geosci. Remote Sens., 43(8), pp. 1699-1706.

Yajima, Y., Yamaguchi, Y., and Sato, R., et al., 2008. POLSAR image analysis of wetlands using a modified four-component scattering power decomposition. IEEE Trans. Geosci. Remote Sens., 46(6), pp. 1667-1673.
Yamaguchi, Y., Sato, A., and Boerner, W. M., et al., 2011. Four-component scattering power decomposition with rotation of coherency matrix. IEEE Trans. Geosci. Remote Sens., 49(6), pp. 2251-2258. 